\title{
Kinetics of Block Copolymer Phase Segregation During Sub-Millisecond Transient Thermal
}

\section{Annealing}

\author{
Alan G. Jacobs, ${ }^{*}{ }^{\dagger}$ Clemens Liedel, ${ }^{\dagger}{ }^{\ddagger}$ Hui Peng, ${ }^{\dagger}$ Linxi Wang, $^{\dagger}$ Detlef-M. \\ Smilgies, "Christopher K. Ober, ${ }^{\dagger}$ and Michael O. Thompson*,† \\ $\dagger$ †epartment of Materials Science $\& 3$ Engineering, Bard Hall, Cornell University, Ithaca, \\ New York 14853 \\ $\ddagger$ Department of Colloid Chemistry, Max Planck Institute of Colloids and Interfaces, \\ Research Campus Golm, 14476 Potsdam, Germany \\ ฯ Cornell High Energy Synchrotron Source (CHESS), Wilson Laboratory, Cornell \\ University, Ithaca, New York 14853 \\ E-mail: agj27@cornell.edu; mot1@cornell.edu
}

\section{Supporting Information Available}

\section{Laser Spike Annealing (LSA)}

Millisecond thermal anneals were achieved by scanning a line-focused continuous wave $\mathrm{CO}_{2}$ laser $(\lambda=10.6 \mu \mathrm{m}, \sim 90 \mu \mathrm{m}$ by $500 \mu \mathrm{m}$ FWHM focus) as shown in Figure S1a. Figure S1b schematically shows the $\mu$-GISAXS measurement geometry relative to the LSA spatial temperature profile. Example measured time-temperature profiles for $10 \mathrm{~ms}$ and $250 \mu \mathrm{s}$ LSA anneals to a peak temperature of $500^{\circ} \mathrm{C}$ are shown in Figure S1c. The LSA anneal duration is measured by the characteristic laser dwell time, $\tau$, defined as the beam FWHM in the 
scan direction (y- direction in Figure S1a) divided by the scan velocity. Thermistor measurements indicate that the sample remains within $\sim 5 \%$ of the peak annealing temperature for approximately $1 \tau$ followed by rapid quench which approaches room temperature after approximately $10 \tau$.
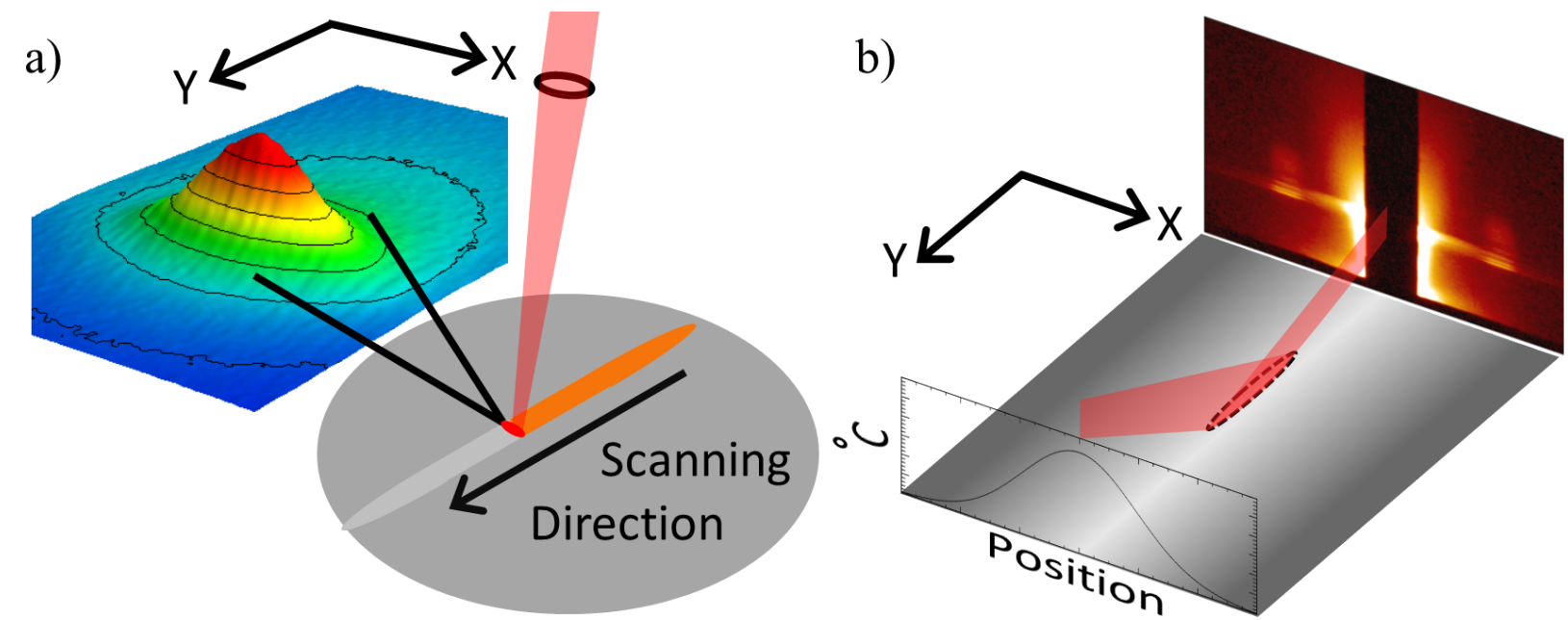

c)

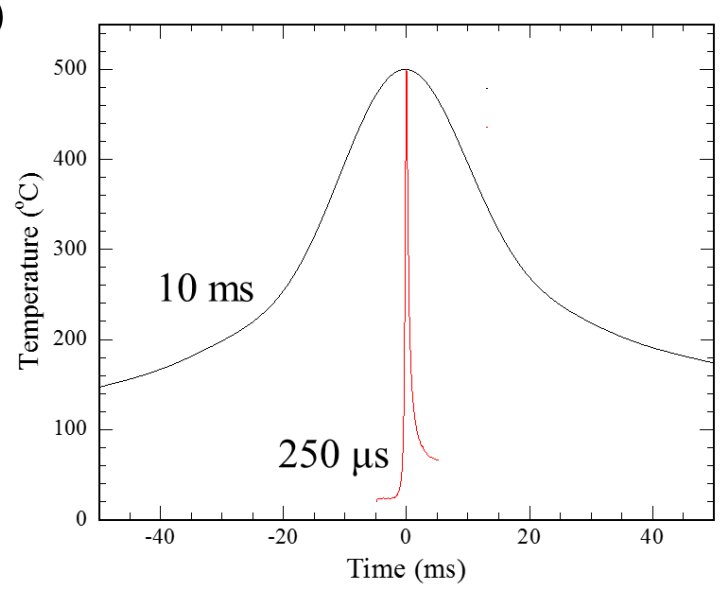

d)

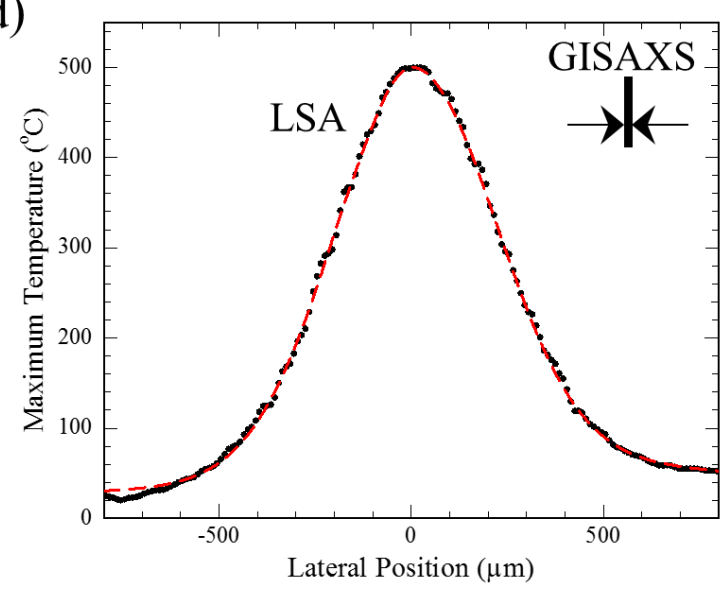

Figure S1: (a) Schematic of LSA geometry with a measured temperature profile in the heated zone. (b) Scale representation of GISAXS probe geometry on lgLSA area, GISAXS FWHM in $\mathrm{x}$ - is $\sim 20 \mu \mathrm{m}$, in $\mathrm{y}-\mathrm{FWHM}$ is $\sim 8 \mathrm{~mm}$. (c) Time-temperature profiles of long $(10 \mathrm{~ms})$ and short $(250 \mu \mathrm{s})$ duration anneals to a peak temperature of $500{ }^{\circ} \mathrm{C}$, and (d) $250 \mu \mathrm{s}$ LSA spatial maximum temperature profile in the $\mathrm{x}$ - direction and approximate GISAXS probe width.

Thin film platinum thermistor measurements were obtained for each dwell to yield temperature maps as shown in Figure S1a. Additionally, these spatial profiles were taken at 
several peak temperatures as the spatial profile is slightly temperature dependent due to changes in silicon thermal diffusivity with temperature. This yields quantitative data for the peak temperature as a function of lateral position ( $\mathrm{x}$ - direction) and as a function of the peak temperature. Figure S1d shows one measurement of the maximum temperature experienced as a function of lateral position across a $250 \mu \mathrm{s}$ LSA. These data were then fit to a sum of Gaussian profiles for conversion from position to peak anneal temperature.

In order to determine accurate temperatures, the center of the LSA scan must be precisely determined. Manual alignment was used to register the $\mu$-GISAXS beam with the scan, with alignment typically better than 100 microns. Spatial temperature profiles were then laterally shifted to ensure overlay of the measured properties as a function of temperature from both sides of the spatial temperature profile. This provides a very sensitive internal alignment reference resulting in overall estimated temperature uncertainties of $\pm 18^{\circ} \mathrm{C}$ including both peak temperature and alignment errors.

\section{Temperature Metric Discussion}

Due to the ex-situ measurement conditions and inherently transient nature of LSA, the specific LSA temperature used as identification of the ODT must be carefully analyzed. Multiple choices (peak temperature, average temperature, half-maximum temperature, etc.) are potential representative values with no single value immediately identifiable as most appropriate to represent the dynamic sample environment, especially after the quench to room temperature.

Thermal diffusion calculations show that a $100 \mathrm{~nm}$ thick film requires of order $10 \mathrm{mi}-$ croseconds to thermally equilibrate $\left(\sqrt{D t} \sim 300 \mathrm{~nm}\right.$ for $\left.\mathrm{D}=10^{-4} \mathrm{~cm}^{2} / \mathrm{s}\right)$, meaning the sample is not thermal diffusion limited and experiences the full time-temperature thermal profile. Mass transport, or any kinetic process which accelerates with temperature, will be inherently more sensitive to the highest temperatures reached during any annealing environment. In the case of Arrhenius kinetics with a reasonable activation energy of $\sim 1 \mathrm{eV}$, integration of 
the LSA time-temperature history (Figures 1a,S1c) shows an equivalent isothermal anneal (infinite heating/quench rates) would be between 0.9 and 1.1 dwell times at the peak temperature. Though not all processes in polymers exhibit Arrhenius behavior, it is important to note that the dominance of Arrhenius diffusion kinetics at high temperatures has been previously shown in some systems ${ }^{1}$.

For the specific case of segregation, the disordering behavior should be most sensitive to the highest temperature achieved rather than some average temperature as any time spent above $\mathrm{T}_{\text {ODT }}$ will initially disorder the material, while quenching just below $\mathrm{T}_{\text {ODT }}$ with partially ordered/disordered films will asymptotically approach the ordered state again. This will favor quenched morphologies in between the fully ordered and fully disordered states. For these reasons, the maximum LSA temperature is reported.

\section{$\mu$-GISAXS Alignment to LSA}

Alignment of the LSA scan direction to the $\mu$-GISAXS measurement beam was performed using a sample microscope and confirmed via visible changes on sample films after periodic long duration exposures. A fluorescence image of the microbeam was obtained from a polished cadmium tungstate substrate and noted on an in-situ microscope image (as discussed by Li et $a l .^{2}$ ). Specially annealed LSA burns were then aligned to the calibrated microscope image via a discernible contrast at the burn edges. Finally, acceptable alignment was confirmed on periodic samples by over-exposing $(>1000 \mathrm{x}$ measurement dose) pristine sample areas near the burn edges to induce visible polymer damage exhibiting parallel geometry.

\section{Thermal Diffusivity Estimation}

From Fickian diffusivity with cylindrical geometry ${ }^{3}$, the necessary thermal dose to perturb a cylinder center concentration by $10 \%$ is $\mathrm{Dt} / \mathrm{r}^{2} \approx 0.085$. The effective diffusivity required

to modify the PMMA cylinders for a particular dwell time is then simply $\mathrm{D}=0.085 \mathrm{r}^{2} / \tau_{\text {dwell }}$. For a cylinder to cylinder spacing of $27.3 \mathrm{~nm}$, the approximate cylinder radius was estimated 
at $6.8 \mathrm{~nm}$ yielding a required diffusivity of $3.96 \times 10^{-11}$ and $1.58 \times 10^{-10} \mathrm{~cm}^{2} / \mathrm{s}$ for $1 \mathrm{~ms}$ and $250 \mu \mathrm{s}$ dwell times, respectively. Though appropriate for a first order approximation, this estimation neglects the potentially significant effects from concentration/chemical potential dependent diffusivity.

\section{Phase Map Temperature Identification}

Figure S2a shows scattering intensity as a function of the peak annealing temperature for the $250 \mu \mathrm{s} \mathrm{LSA}$. The boundaries for the phase map were determined as the intersection of linear extrapolation within each temperature regime. Figure S2b shows the traditionally used reciprocal intensity method which identifies an effective kinetic disordering temperature, $\mathrm{T}_{\mathrm{ODT}}$,eff, between the onset of decreased signal intensity and the history independent temperature. Figures S3-S6 show data for $500 \mu$ s to $10 \mathrm{~ms}$ respectively.

Similarly, Figure S7 shows the identification of $\mathrm{T}_{\mathrm{ODT}}$ for hot plate anealing. Due to the slow heating rate $\left(1^{\circ} \mathrm{C} / \mathrm{min}\right)$, these data exhibit a much steeper slope and hence a well-defined $\mathrm{T}_{\mathrm{ODT}}$. In contrast, LSA data show much more gradual changes in the final scattering intensity, and the $\mathrm{T}_{\mathrm{ODT}}$,eff extracted by this method becomes less precise. In lieu of the traditional inverse scattering intensity method, the first onset of GISAXS scattering intensity loss was taken as indication of the onset of disordering $\left(\mathrm{T}_{\text {disorder }}\right)$. The identified temperatures are summarized in Table S1 and were used to create the phase maps. Note that the uncertainty in the intersection temperature listed in Table S1 does not include the additional $\pm 18^{\circ} \mathrm{C}$ systematic temperature uncertainty for these LSA data. Figure S8 shows the phase maps from the identified temperatures in Table S1. Figure S8a includes the additional identified $\mathrm{T}_{\mathrm{ODT}}$,eff which shows a different absolute disordering temperature but follows the same trend.

It should be noted that although all films remained stable for all conditions as measured by film thickness loss, distinct damage is apparent for $1 \mathrm{~ms}$ and longer anneals at the highest temperatures where GISAXS scattering intensity signal is lost. This degradation did not 
impact identification of transition temperatures as it occurred beyond the region of interest for all anneals.

a)

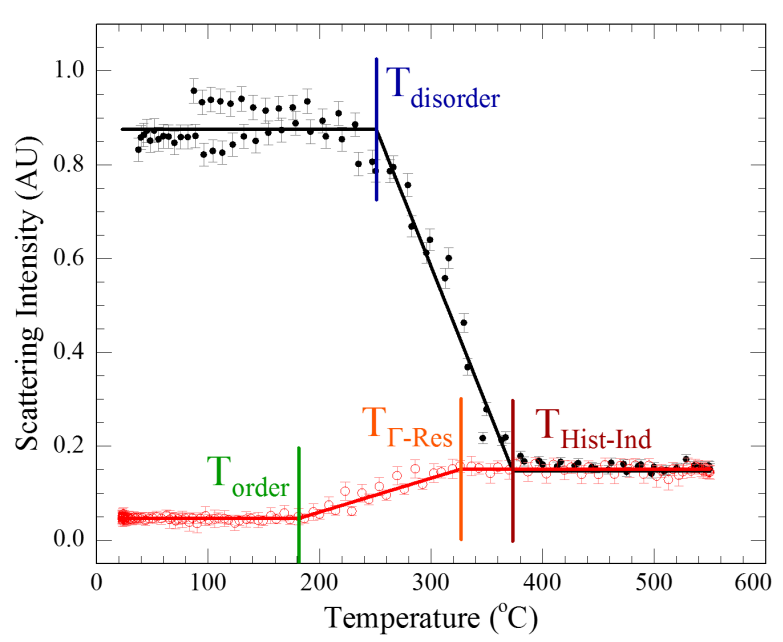

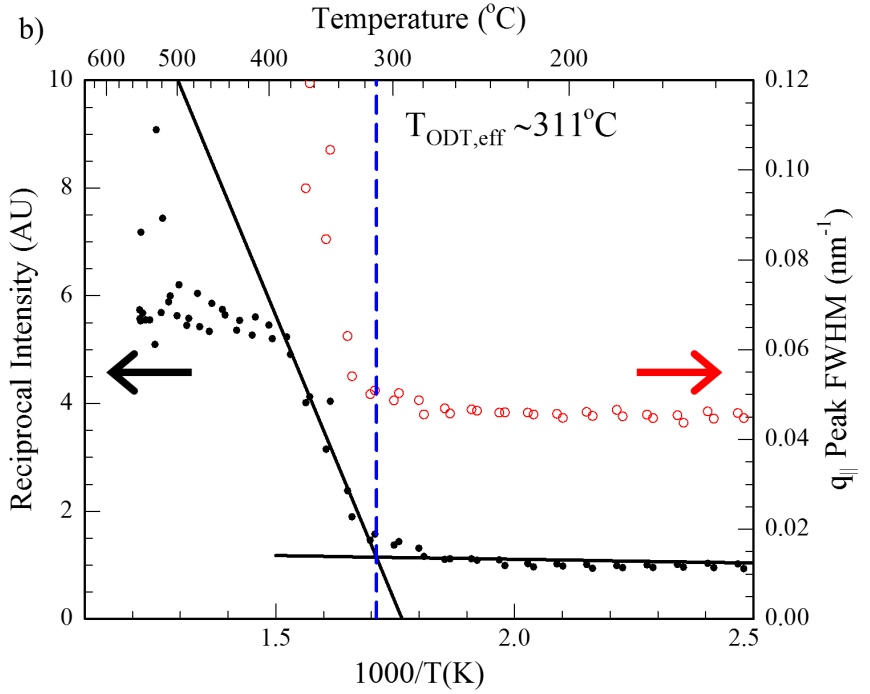

Figure S2: Identification of critical temperatures by linear extrapolation for the $250 \mu \mathrm{s}$ LSA for (a) $\mathrm{T}_{\text {disorder }}, \mathrm{T}_{\text {History-Independence, }}, \mathrm{T}_{\text {order }}, \mathrm{T}_{\Gamma_{\text {residual }}}$, and (b) $\mathrm{T}_{\text {ODT-effective }}$.

Table S1: Summary of critical temperatures identified for phase segregation and disordering.

\begin{tabular}{|c|c|c|c|c|c|c|}
\hline Anneal Duration & $\begin{array}{c}\mathrm{T}_{\text {disorder }} \\
\pm 2^{\circ} \mathrm{C}\end{array}$ & $\begin{array}{c}\mathrm{T}_{\text {ODT,eff }} \\
\pm 5^{\circ} \mathrm{C}\end{array}$ & $\begin{array}{c}\mathrm{T}_{\text {Hist-Ind }} \\
\pm 3{ }^{\circ} \mathrm{C}\end{array}$ & $\begin{array}{l}\mathrm{T}_{\text {order }} \\
\pm 3^{\circ} \mathrm{C}\end{array}$ & $\begin{array}{c}\mathrm{T}_{\Gamma_{\text {residual }}} \\
\pm 3{ }^{\circ} \mathrm{C}\end{array}$ & $\Gamma_{\text {residual }}$ \\
\hline $250 \mu \mathrm{s}$ & 251 & 311 & 373 & 181 & 326 & 0.171 \\
\hline $500 \mu \mathrm{s}$ & 215 & 283 & 351 & 142 & 275 & 0.245 \\
\hline $1 \mathrm{~ms}$ & 189 & 249 & 306 & 120 & 223 & 0.298 \\
\hline $2 \mathrm{~ms}$ & 191 & 244 & 318 & 119 & 221 & 0.355 \\
\hline $10 \mathrm{~ms}$ & 194 & 210 & 279 & $<128$ & 189 & 0.448 \\
\hline Hot Plate & $\mathrm{T}_{\text {ODT }}$ & $179 \pm 2{ }^{\circ} \mathrm{C}$ & - & $\mathrm{T}_{g}$ & - & $<0.025$ \\
\hline
\end{tabular}

This material is available free of charge via the Internet at http://pubs.acs.org/. 

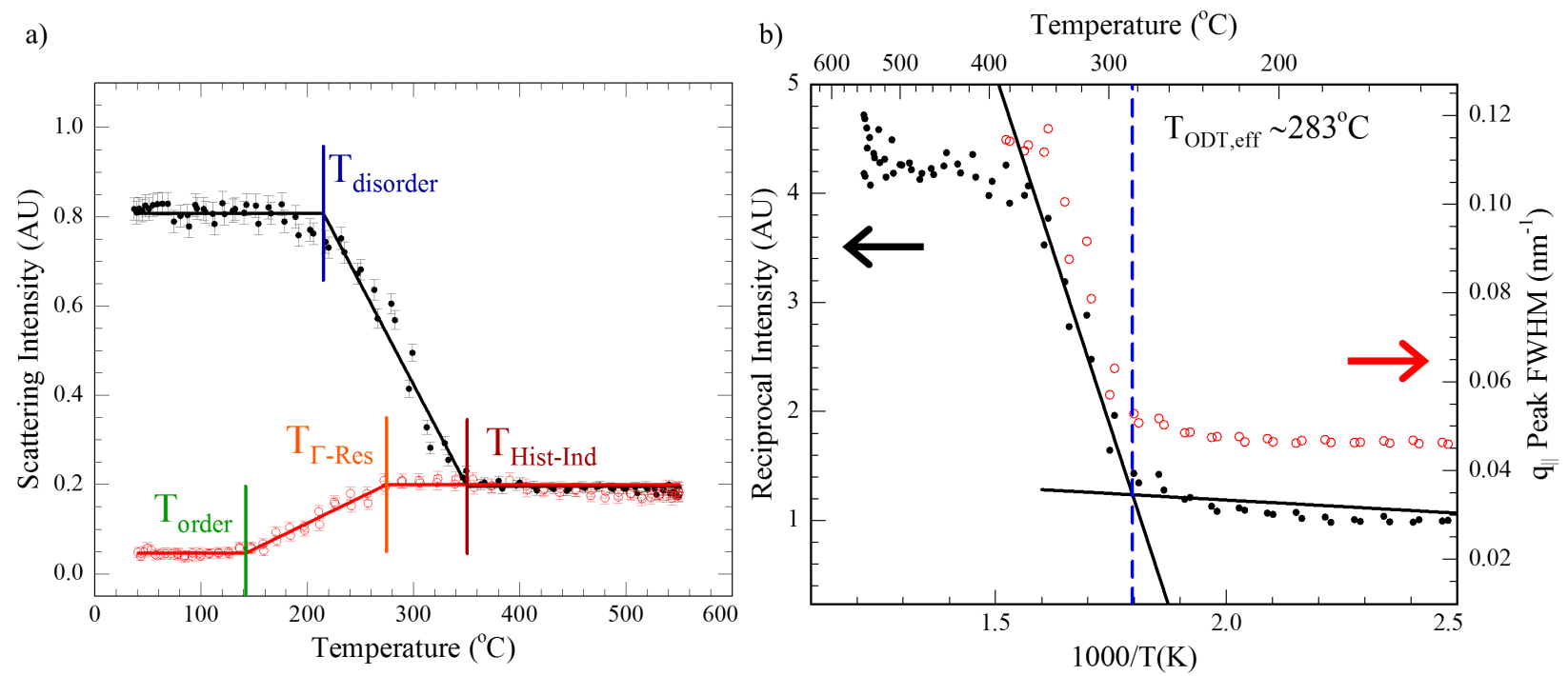

Figure S3: Identification of critical temperatures by linear extrapolation for the $500 \mu \mathrm{s}$ LSA for (a) $\mathrm{T}_{\text {disorder }}, \mathrm{T}_{\text {History-Independence, }}, \mathrm{T}_{\text {order }}, \mathrm{T}_{\Gamma_{\text {residual }}}$, and (b) $\mathrm{T}_{\text {ODT-effective }}$.
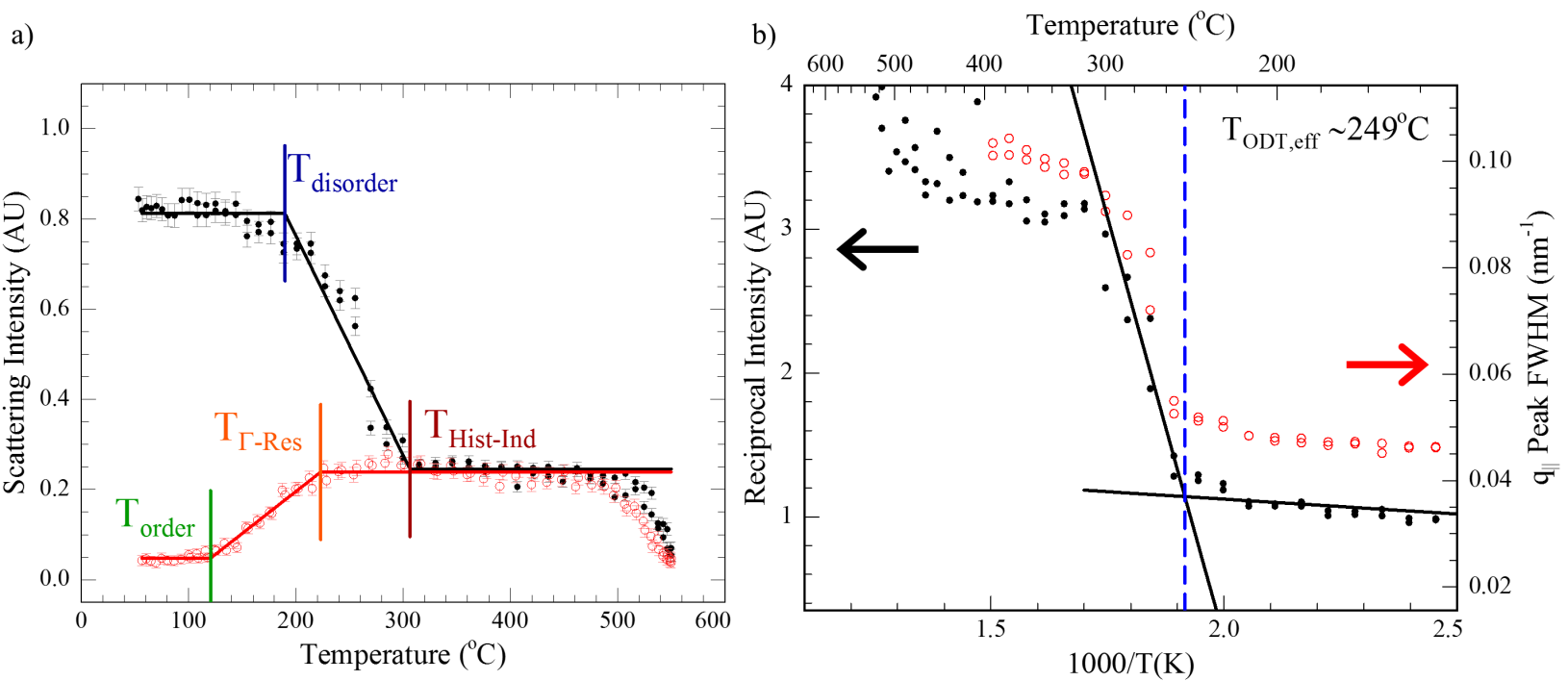

Figure S4: Identification of critical temperatures by linear extrapolation for the $1 \mathrm{~ms}$ LSA for (a) $\mathrm{T}_{\text {disorder }}, \mathrm{T}_{\text {History-Independence, }} \mathrm{T}_{\text {order }}, \mathrm{T}_{\Gamma_{\text {residual }}}$, and (b) $\mathrm{T}_{\text {ODT-effective }}$. 
a)

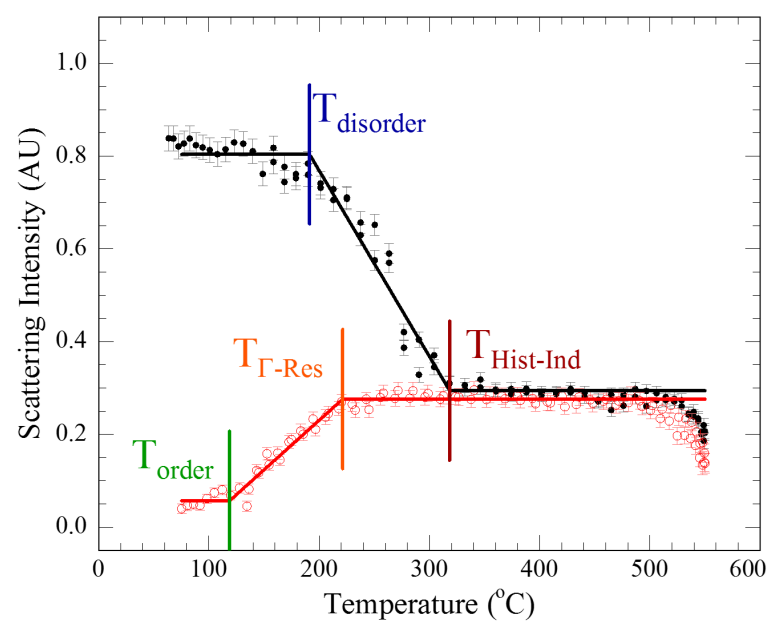

b) Temperature $\left({ }^{\circ} \mathrm{C}\right)$

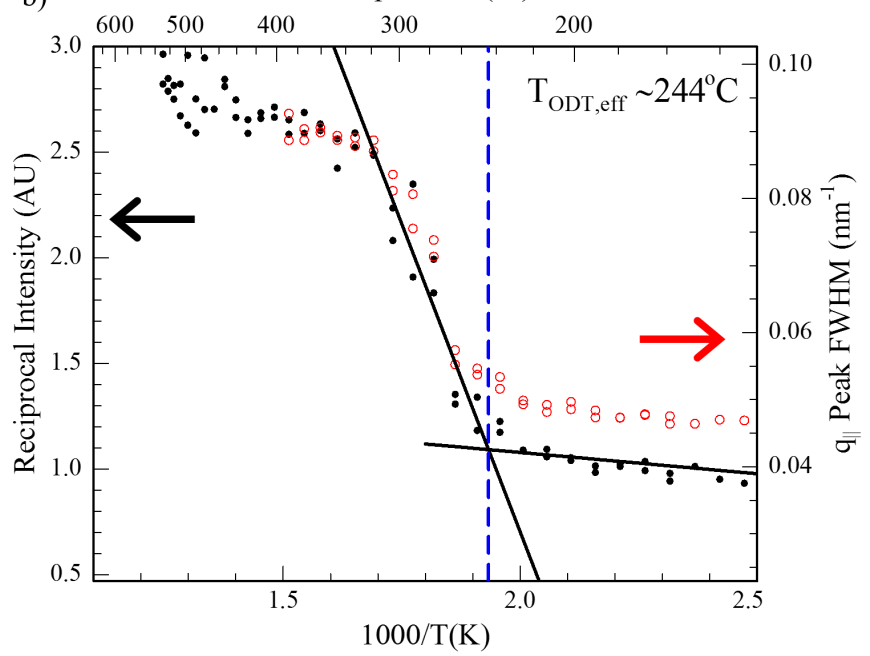

Figure S5: Identification of critical temperatures by linear extrapolation for the $2 \mathrm{~ms}$ LSA for (a) $\mathrm{T}_{\text {disorder }}, \mathrm{T}_{\text {History-Independence }}, \mathrm{T}_{\text {order }}, \mathrm{T}_{\Gamma_{\text {residual }}}$, and (b) $\mathrm{T}_{\mathrm{ODT}-\text { effective }}$.

a)

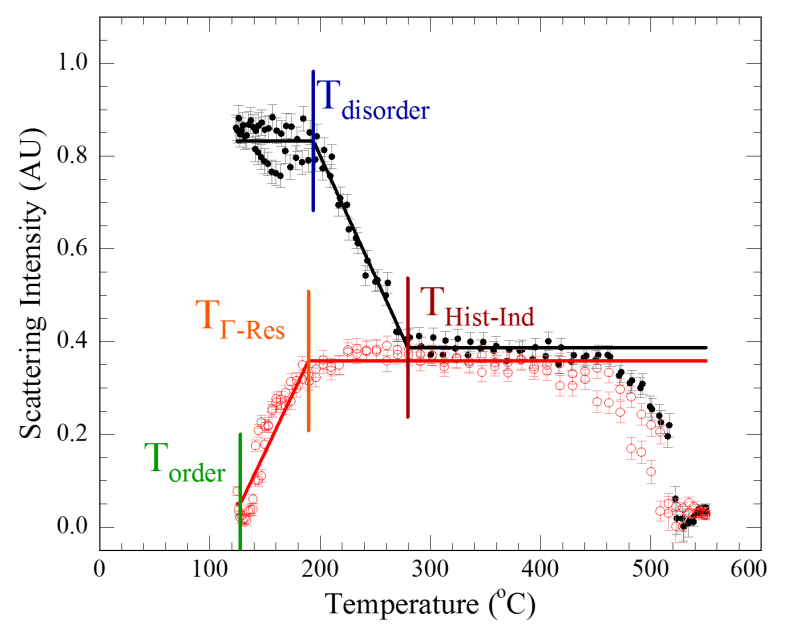

b) Temperature $\left({ }^{\circ} \mathrm{C}\right)$

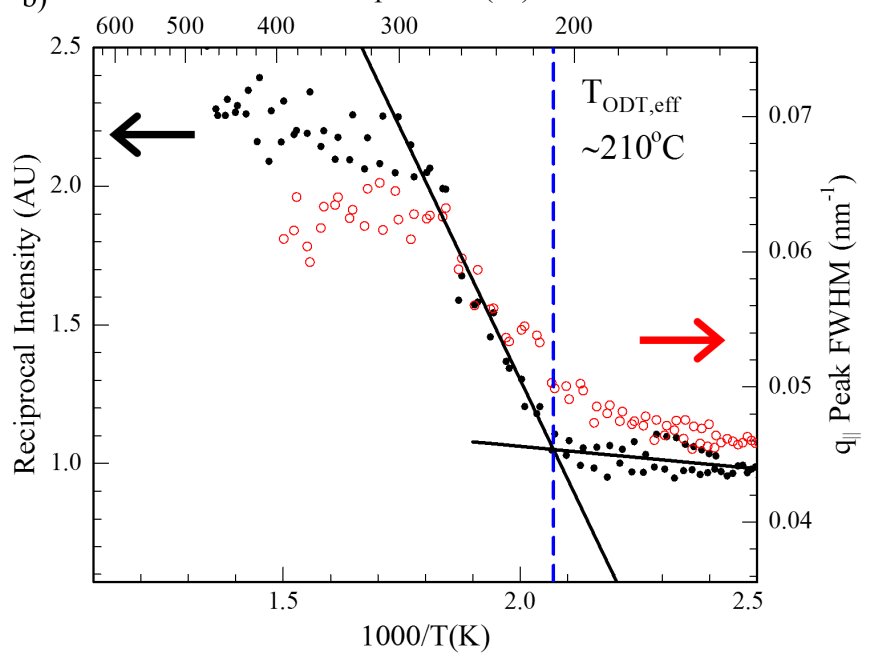

Figure S6: Identification of critical temperatures by linear extrapolation for the $10 \mathrm{~ms}$ LSA for (a) $\mathrm{T}_{\text {disorder }}, \mathrm{T}_{\text {History-Independence }}, \mathrm{T}_{\text {order }}, \mathrm{T}_{\Gamma_{\text {residual }}}$, and (b) $\mathrm{T}_{\mathrm{ODT} \text {-effective }}$. 


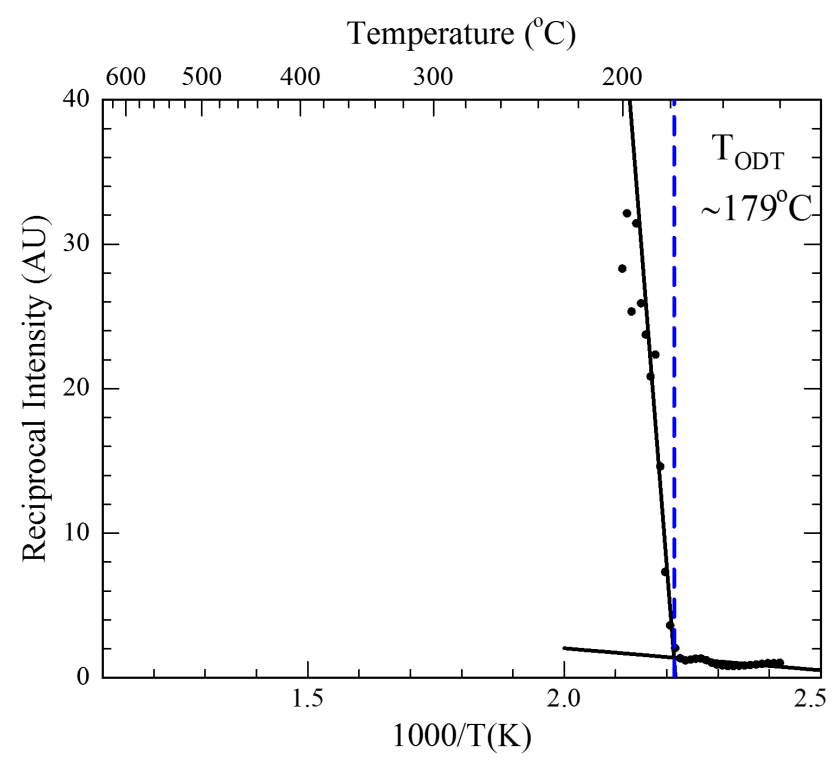

Figure S7: Identification of $\mathrm{T}_{\mathrm{ODT}}$ for in-situ hot plate annealing.
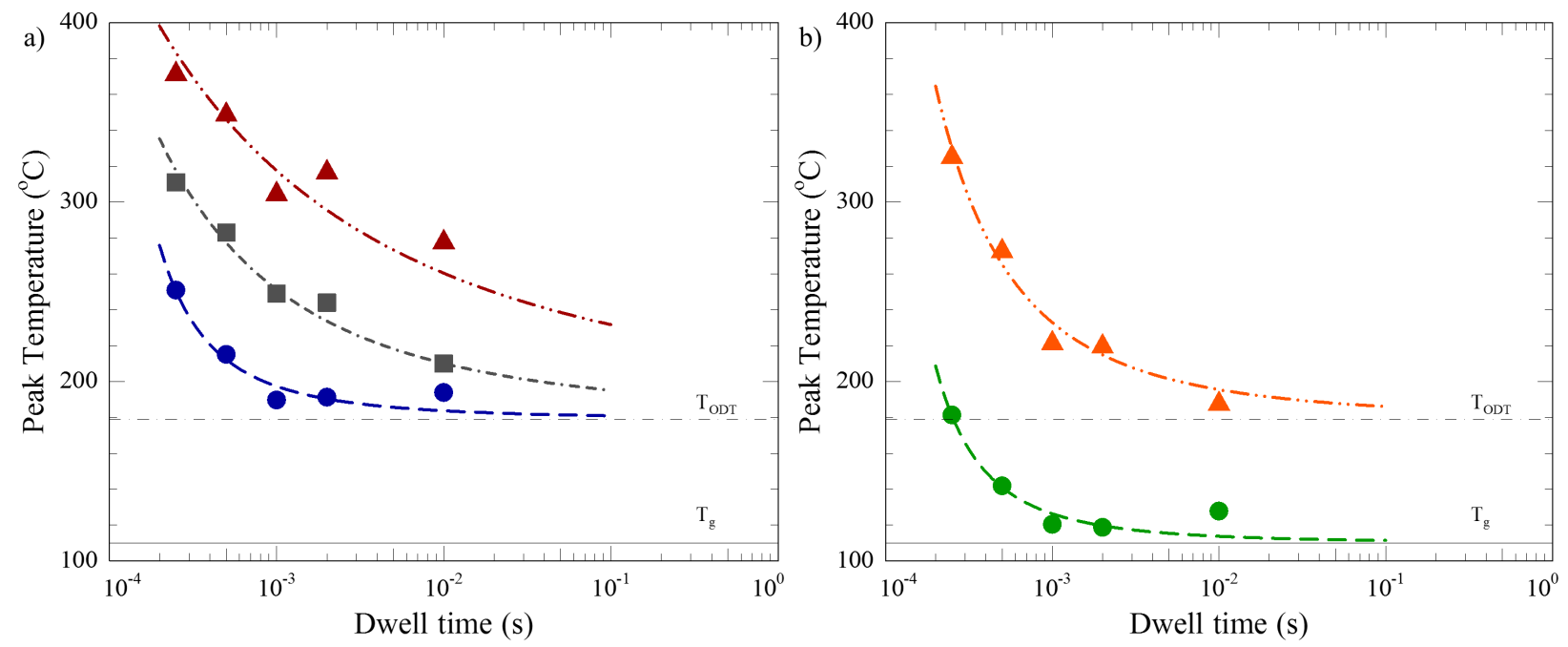

Figure S8: Phase map after lgLSA from (a) initially phase segregated material showing $\mathrm{T}_{\text {disorder }}$ (dashed curve, blue circles), $\mathrm{T}_{\mathrm{ODT} \text {,eff }}$ (dash-dash-dot curve, grey squares), and $\mathrm{T}_{\text {Hist-Ind }}$ (dash-dot-dot curve, red triangles). (b) Phase map from intially disordered, asspun material showing $\mathrm{T}_{\text {order }}$ (dashed curve, green circles) and $\mathrm{T}_{\Gamma_{\text {residual }}}$ (dash-dot-dot curve, orange triangles). Lines to guide the eye only. 

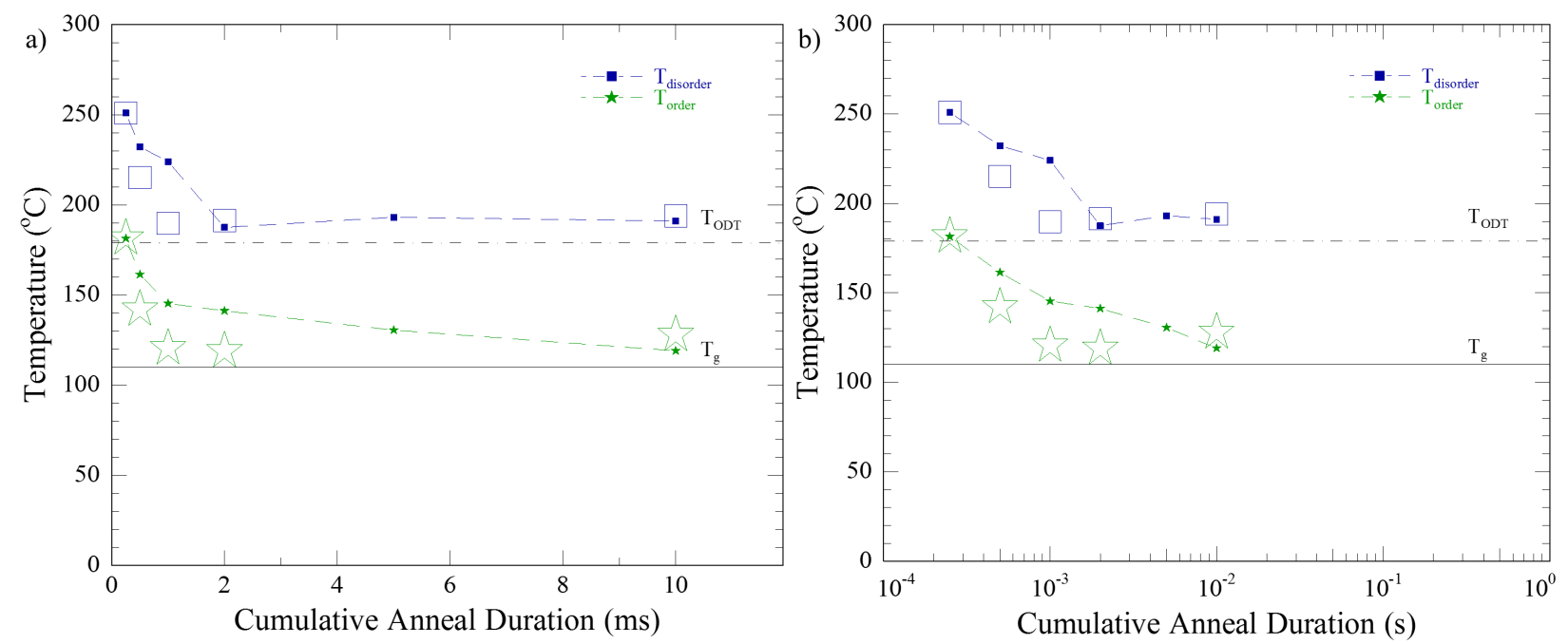

Figure S9: Time dependence of disordering and ordering onset temperatures for consecutive $250 \mu$ s anneals (closed symbols, line) compared to single LSA anneals of longer duration (open symbols) shown on (a) linear time scales and (b) log time scales. 


\section{References}

(1) Jung, B.; Sha, J.; Paredes, F.; Chandhok, M.; Younkin, T. R.; Wiesner, U. B.;

Ober, C. K.; Thompson, M. O. Kinetic rates of thermal transformations and diffusion in polymer systems measured during sub-millisecond laser-induced heating. ACS Nano 2012, 6, 5830-5836.

(2) Li, R.; Ward, J. W.; Smilgies, D.-M.; Payne, M. M.; Anthony, J. E.; Jurchescu, O. D.; Amassian, A. Direct Structural Mapping of Organic Field-Effect Transistors Reveals Bottlenecks to Carrier Transport. Advanced Materials 2012, 24, 5553-5558.

(3) Crank, J. The Mathematics of Diffusion, 2nd ed.; Oxford University Press, 1975. 